\title{
Gamma Knife Radiosurgery for Vestibular Schwannomas: Results of Hearing Preservation in Relation to the Cochlear Radiation Dose
}

Ferdinand C. A. Timmer, MD; Patrick E. J. Hanssens, MD, PhD; Anniek E. P. van Haren, MD; Jef J. S. Mulder, MD, PhD; Cor W. R. J. Cremers, MD, PhD; Andy J. Beynon, PhD; Jacobus J. van Overbeeke, MD, PhD; Kees Graamans, MD, PhD

Objectives/Hypothesis: This study was designed to evaluate hearing preservation after gamma knife radiosurgery (GKRS) and to determine the relation between hearing preservation and cochlear radiation dose in patients with a sporadic vestibular schwannoma (VS).

Methods: Prospective study involving patients suffering from VS who received GKRS from June 2003 until November 2007. Pure tone and speech audiometry were conducted before and after GKRS. The thresholds at pure tone audiometry were taken as a measure of hearing. Pure tone average (PTA) was defined as the mean threshold at $0.5 \mathrm{kHz}, 1.0 \mathrm{kHz}$, $2.0 \mathrm{kHz}$, and $4.0 \mathrm{kHz}$. Hearing was classified according to the 2003 consensus meeting in Tokyo. Stereotactic surgery was performed using a Leksell 4C Gamma Knife (Elekta, Stockholm, Sweden).

Results: A total of 69 patients were included in the study. Mean tumor size was $17 \mathrm{~mm}$. Mean marginal dose at the tumor was 11.0 Gy (range, 9.3 Gy$12.3 \mathrm{~Gy}$ ), mean maximal dose was 19.7 Gy (range, 16 Gy-25.5 Gy). Mean maximal dose at the cochlea was 10.27 Gy (range, 3.1 Gy-16.1 Gy), and mean minimal dose at the cochlea was 2.6 Gy (range, 0.9 Gy-7.4 Gy). Mean PTA before GKRS was $43 \mathrm{~dB}$ (standard deviation [SD] $20 \mathrm{~dB}$ ), mean PTA after GKRS was 63

From the Radboud University Nijmegen Medical Center, Donders Institute for Brain, Cognition and Behavior, Department of Otorhinolaryngology, Head and Neck Surgery, Nijmegen, The Netherlands (F.C.A.T., A.E.P.v.H., J.J.s.M., C.w.R.J.C., A.J.B., K.G.); and Gamma Knife Center Tilburg, St. Elisabeth Hospital, Tilburg, The Netherlands (P.E.J.H., J.J.v.o.)

Editor's Note: This Manuscript was accepted for publication February 9, 2009.

Send correspondence to Ferdinand C. A. Timmer, Radboud University Nijmegen Medical Centre, Donders Institute for Brain, Cognition and Behaviour, Department of Otorhinolaryngology \& Head and Neck Surgery, PO BOX 9101, 6500 HB Nijmegen, The Netherlands. E-mail: f.timmer@kno.umcn.nl

DOI: 10.1002/lary.20245
$\mathrm{dB}$ (SD $30 \mathrm{~dB}$ ). Mean interval between pre-GKRS audiometry and GKRS was 8.0 months. Between GKRS and post-GKRS audiometry, mean interval was 14.2 months. Hearing was considered to be preserved (max +1 class, Tokyo classification) in $52(75 \%)$ of 69 patients. However, only 32 patients had class A, B, or C (serviceable hearing) before GKRS. Within this group, only 13 patients $(41 \%)$ had a hearing class A, $\mathrm{B}$, or C after GKRS. A significant relation was found between the maximal cochlear dose and the difference in PTA before and after GKRS.

Conclusions: Hearing preservation is correlated to the maximal radiation dose at the cochlea. The purpose of developing GKRS techniques was to avoid collateral damage in healthy tissues. This study emphasizes the need for exact radiation planning to reduce the cochlear radiation dose if the hearing is to be preserved.

Key Words: Acoustic neuroma, audiometry, hearing preservation, gamma knife, radiosurgery, complications, vestibular schwannoma.

Laryngoscope, 119:1076-1081, 2009

\section{INTRODUCTION}

Due to the increased availability of magnetic resonance imaging (MRI), vestibular schwannomas (VSs) are discovered regularly and diagnosed more frequently at an earlier stage. ${ }^{1-3}$ Initial treatment strategies include conservative management (wait-and-scan), microsurgery, and radiation therapy. Radiation therapy can be subdivided into fractionated radiotherapy and radiosurgery. Leksell initially introduced stereotactic methods and radiosurgery in $1951 .{ }^{4}$ The first VSs were treated by gamma knife radiosurgery (GKRS) in $1971 .{ }^{5}$ Nowadays, GKRS consists of administering a single dose of radiation after 3D MRI and stereotactic planning using a fixed frame on the head.

Besides being the most common presenting symptom, sensorineural hearing loss is considered a risk 
factor in both radiotherapy and microsurgery management of VS. Obviously, translabyrinthine surgery causes deafness and is the least attractive option if hearing is to be preserved. Other microsurgical approaches can preserve some hearing, although the results vary among individual surgeons and the surgical approach chosen.

Due to the relatively mild risk profile, the comfort provided to the patient, and the high tumor control rate of GKRS, an increasing percentage of our VS patients prefer primary treatment with GKRS above microsurgery. Hearing loss resulting from cochlear and cochlear nerve radiation damage is frequently observed after GKRS, although the complication rate is low.

The aim of this study is to evaluate our results for hearing preservation after GKRS. In that light, it then explores the relation between hearing preservation and cochlear radiation dose. This is done by finding correlations between radiation dose at both tumor and cochlea on the one hand, and hearing preservation on the other.

\section{MATERIALS AND METHODS}

\section{Study Group}

All patients were initially seen and examined at the Department of Otorhinolaryngology, Head and Neck Surgery of the Radboud University Nijmegen Medical Center. Between May 2003 and November 2007, 111 patients were referred for radiosurgery to the Gamma Knife Center in Tilburg. These patients had tumors $<3.0 \mathrm{~cm}$ at the first scan. They were referred either because of MRI-proven tumor growth $(>2 \mathrm{~mm}$ maximal diameter of the tumor in either direction on axial MRI) or because of their personal preference. Patients suffering from neurofibromatosis and patients with a pure tone average (PTA) $>90 \mathrm{~dB}$ before GKRS were excluded from this study.

\section{Tumor Location and Size}

Two individual observers performed the assessments of the tumor size. The continuation of the line of the petrous ridge depicted on serial axial MRI scans provided a clear distinction between the intra- and extrameatal portion of the tumor. The tumor was reported as an intrameatal lesion when there was no tumor extension beyond that plane. Tumor size was determined according to the consensus reached in Tokyo on November 7, 2003. ${ }^{6}$ This measurement only includes the largest extrameatal diameter. Measurements, according to the guidelines of the American Academy of Otolaryngology-Head and Neck Surgery, were performed as well to compare results. ${ }^{7}$ In the latter method, size is the square root of the product between the greatest diameter in the direction parallel to the petrous ridge and the maximum diameter perpendicular to this in the axial plain. Furthermore, it was noted whether the fundus was filled with tumor or empty. An empty fundus showed a high signal behind the tumor in the fundus on a T2-weighted MRI scan.

\section{Audiometry}

Pure tone audiometry was performed before and after GKRS. The PTA of bone conduction was assessed for both ears. PTA was defined as the mean of the thresholds at $0.5 \mathrm{kHz}$, $1.0 \mathrm{kHz}, 2.0 \mathrm{kHz}$, and $4.0 \mathrm{kHz}$. When no threshold could be measured, it was noted at $130 \mathrm{~dB}$ hearing loss.

The maximum speech discrimination score (SDS) was determined by speech audiometry measurements with Dutch
TABLE I.

Hearing Classification Consensus Meeting, Tokyo 2003. ${ }^{6}$

\begin{tabular}{lcr}
\hline Class & PTA (dB) & SDS (\%) \\
\hline A & $0-20$ & $100-80$ \\
B & $21-30$ & $79-70$ \\
C & $31-40$ & $69-60$ \\
D & $41-60$ & $59-50$ \\
E & $61-80$ & $49-40$ \\
F & $>80$ & $39-0$ \\
\hline \hline
\end{tabular}

PTA $=$ pure tone average; SDS $=$ speech discrimination score

vowel-consonant-vowel words up to a maximum of $125 \mathrm{~dB}$ sound pressure level.

Hearing was classified according to the 2003 consensus meeting in Tokyo. ${ }^{6}$ This classification is summarized in Table I.

\section{Gamma Knife Settings, Procedure, and Cochlear Radiation Dose}

A Leksell titanium stereotactic frame (Elekta, Stockholm, Sweden) was installed on each patient's head after injecting local anesthesia subcutaneously above the screw points in the skull. The patient was then placed in a Philips T1.0 MRI scanner (Philips Healthcare, Andover, MA). Axial T1 1.0 mm MRI images were made before and after administering gadolinium contrast. Then 3D TSE $0.7 \mathrm{~mm}$ images were produced.

Using Leksell Gamma Plan software V5.34 (Elekta), the tumor was delineated on the corresponding images using the TSE- and T1-weighted gadolinium contrast enhanced images. The tumor volume was then calculated using the $3 \mathrm{D}$ software. A radiation plan was made by placing isocenters or shots in the tumor volume, guided by prescribed dose (PD), marginal dose, and conformity. The radiation dose was prescribed to the isodose covering $90 \%$ of the tumor volume. In patients who were still able to use their affected ear on the telephone, a dose of 12.5 Gy was prescribed. The PD for patients who declared their affected ear to be useless was 13.0 Gy. The resulting marginal dose was on average 11.0 Gy for all patients (range, 9.3 Gy-12.5 Gy).

The cochlea was pointed out on each axial MRI image, and its volume was also calculated using the same gamma-plan software. In the same procedure the maximum, 50\%, 90\%, and the minimum radiation dose on the cochlea was calculated (Fig. 1).

Stereotactic surgery was performed using a Leksell 4C Gamma Knife (Elekta). Patients were discharged from the hospital the same day.

\section{Statistics}

The difference in PTA between before and after GKRS was calculated for each patient. Correlations between the cochlear radiation dose and the PTA difference were calculated in SPSS version 14.0 (SPSS, Chicago, IL) using a onetailed Pearson test. Scatter plots and regression lines were also calculated using SPSS. The significance of tumor invasion in the fundus was determined using an independent sample $t$ test.

\section{RESULTS}

Of the initial 111 VS patients who were referred for GKRS, 20 were excluded because their prestereotactic radiosurgery PTA was $>90 \mathrm{~dB}$. Four patients had 


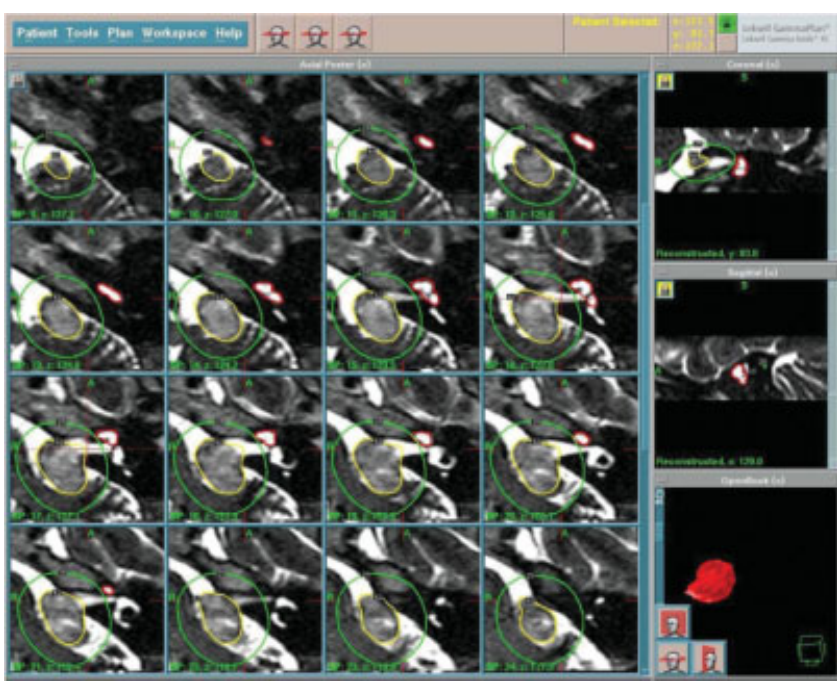

Fig. 1. The limits of the vestibular schwannoma and cochlea are depicted by lines. [Color figure can be viewed in the online issue, which is available at www.interscience.wiley.com.]

neurofibromatosis and were excluded as well. Two patients were not able to participate in examinations because of health problems unrelated to the VS. Sixteen patients were lost to follow-up at the time the control audiogram was scheduled. Eventually 69 patients entered the study.

Forty-four of these patients were referred because of documented tumor growth $(>2 \mathrm{~mm})$ after wait-andscan management. Twenty-two patients were referred for GKRS for various other reasons (e.g., tumor size combined with other factors influencing the initial decision making). Three patients who were initially advised to take part in the wait-and-scan program received GKRS because of their personal preference.

\section{TABLE II.}

Descriptive Statistics of the Study Group.

Sex
Mean age at VS diagnosis,
y (range)
Mean age at SRS, y (range)
Tumor side, right/left
Tumor location, extrameatal/intrame
Fundus IAM, empty/filled
Mean tumor size, Tokyo 2003
guidelines, mm (range)
Mean tumor size AAO-HNS 1995
guidelines, mm (range)
Mean tumor volume, mm³ (range)
38 male/31 female $51(17-73)$

$53(24-76)$ 42 right $(61 \%)$ 27 left (39\%)

66 extrameatal (96\%), 3 intrameatal $(4 \%)$ 40 empty (58\%), 29 filled $(42 \%)$ 17 (6-32)

$17(6-33)$ $2281(24-10,200)$

VS = vestibular schwannoma; SRS = stereotactic radiosurgery; IAM = internal auditory meatus; AAO-HNS = American Academy of Otolaryngology-Head and Neck Surgery.
TABLE III.

Doses in the Tumor.

\begin{tabular}{lccl}
\hline & Mean & Range & SD \\
\hline Dose $90 \%$ & $12.7 \mathrm{~Gy}$ & $12-13.2 \mathrm{~Gy}$ & 0.33 \\
Isodose $90 \%$ & $64 \%$ & $51 \%-75 \%$ & 4.5 \\
Marginal dose & $11.0 \mathrm{~Gy}$ & $9.3-12.5 \mathrm{~Gy}$ & 0.46 \\
Maximal dose & $19.7 \mathrm{~Gy}$ & $16-25.5 \mathrm{~Gy}$ & 1.73 \\
\hline \hline
\end{tabular}

$\mathrm{SD}=$ standard deviation.

Dose $90 \%$ is the minimal amount of grays received by $90 \%$ of the tumor. The marginal dose is the minimal dose in the tumor. The maximal dose is the maximal dose within the tumor.

\section{Descriptive Statistics}

An overview of descriptive statistics is summarized in Table II. The doses in the tumor and cochlea are given in Tables III and IV.

\section{Hearing Preservation}

PTA was calculated before and after GKRS. The mean time between pre-GKRS audio and GKRS was 8.0 months (range, 1 month-31 months). Between the GKRS and post-GKRS audio the mean duration was 14.2 months (range, 3 months-56 months). There was no significant correlation between the PTA difference (= PTA pre-GKRS minus PTA post-GKRS) and the time between GKRS and post-GKRS hearing test $(P=.7)$. The mean PTAs are illustrated in Table V.

The hearing classification before and after GKRS is shown in Figure 2 and and Table VI.

No significant correlation was found between the maximal dose at the tumor and the worsening of hearing $(r=0.16, P=.2)$. However, there was a clear correlation between the maximal cochlear dose and the difference in PTA before and after GKRS (Spearman correlation, $r=$ $0.3, P<.05$, two-tailed test) (Figures 3 and 4 ).

No correlation was found between the tumor volume $(P=.36)$ or size $(P=.29)$ and the PTA difference. If the VS was filling the fundus of the internal auditory canal, this was correlated to a higher PTA difference $(P<.005)$ (Table VII).

A notable finding was the correlation between tumor size and pre-GKRS hearing. Small tumors were correlated with higher PTA $(r=0.3, P<.05)$. See Figure 5.

TABLE IV.

Doses in the Cochlea.

\begin{tabular}{lrll}
\hline & Mean & \multicolumn{1}{c}{ Range } & SD \\
\hline Cochlea min, Gy & 2.6 & $0.9-7.4$ & 1 \\
Cochlea 90\%, Gy & 3.4 & $1.3-8.8$ & 1.1 \\
Cochlea 50\%, Gy & 4.9 & $2.1-10.5$ & 1.55 \\
Cochlea max, Gy & 10.3 & $3.1-16.1$ & 2.9 \\
\hline \hline
\end{tabular}

Cochlea min is the minimal dose within the cochlea. Cochlea $90 \%$ is the minimal amount of grays received by $90 \%$ of the cochlea. Cochlea $50 \%$ is the minimal amount of grays received by $50 \%$ of the cochlea. Cochlea max is the maximal dose within the cochlea. 
TABLE V.

Mean PTA, Range, and the Standard Deviation Before and After GKRS.

\begin{tabular}{lccc}
\hline & $\begin{array}{c}\text { Mean PTA } \\
(\mathrm{dB} \mathrm{HL})\end{array}$ & $\begin{array}{c}\text { Range } \\
(\mathrm{dB} \mathrm{HL})\end{array}$ & SD \\
\hline Tumor ear before GKRS & 43 & $5-90$ & 20 \\
Contralateral ear before GKRS & 18 & $-1-130$ & 19 \\
Tumor ear after GKRS & 63 & $-1-130$ & 31 \\
Contralateral ear after GKRS & 20 & $0-130$ & 20 \\
\hline \hline
\end{tabular}

PTA = pure tone average; GKRS = gamma knife radiosurgery; $\mathrm{dB}$ $\mathrm{HL}=$ decibels hearing level; $\mathrm{SD}=$ standard deviation

\section{Complications}

Five patients reported a temporary decrease in facial muscle function ( $<3$ months). None suffered permanent facial nerve problems. Six patients complained about sensibility disturbances in the face, but in all cases this was transient ( $<3$ months).

\section{DISCUSSION}

In this study we validated the relation between cochlear radiation dose and hearing deterioration in a group of patients treated with a mean marginal tumor dose of $11 \mathrm{~Gy}(9.3 \mathrm{~Gy}-12.5 \mathrm{~Gy})$. We also confirmed the hypothesis that patients with tumors occupying the fundus of the internal auditory canal are at greater risk of hearing loss after GKRS than patients with a fundus free of tumor. Hearing was considered to be preserved $(\max +1$ class, Tokyo classification) in $52(75 \%)$ of the 69 patients. However, just 32 of all the patients had a Tokyo class A, B, or C (serviceable hearing) before GKRS. Within this selected group with a Tokyo class A, B, or C before GKRS, only 13 patients (41\%) had a hearing class $\mathrm{A}, \mathrm{B}$, or $\mathrm{C}$ after GKRS. In other words, when serviceable hearing was present, this could be preserved in only $41 \%$ of the cases.

The pathogenesis of sensorineural hearing loss in patients with VS is still not fully understood. Pressure on the auditory nerve or on the blood vessels supplying the nerve and cochlea might explain part of this process. However, some follow-up studies in patients who had nongrowing tumors also showed a progressive hearing loss. ${ }^{8}$ A possible explanation may lie in ototoxic dispersing tumor metabolites, a process which is not clarified yet. After GKRS, a temporary swelling of the VS can occur. Alongside the higher radiation dose, this swelling could partly explain the greater risk of hearing loss
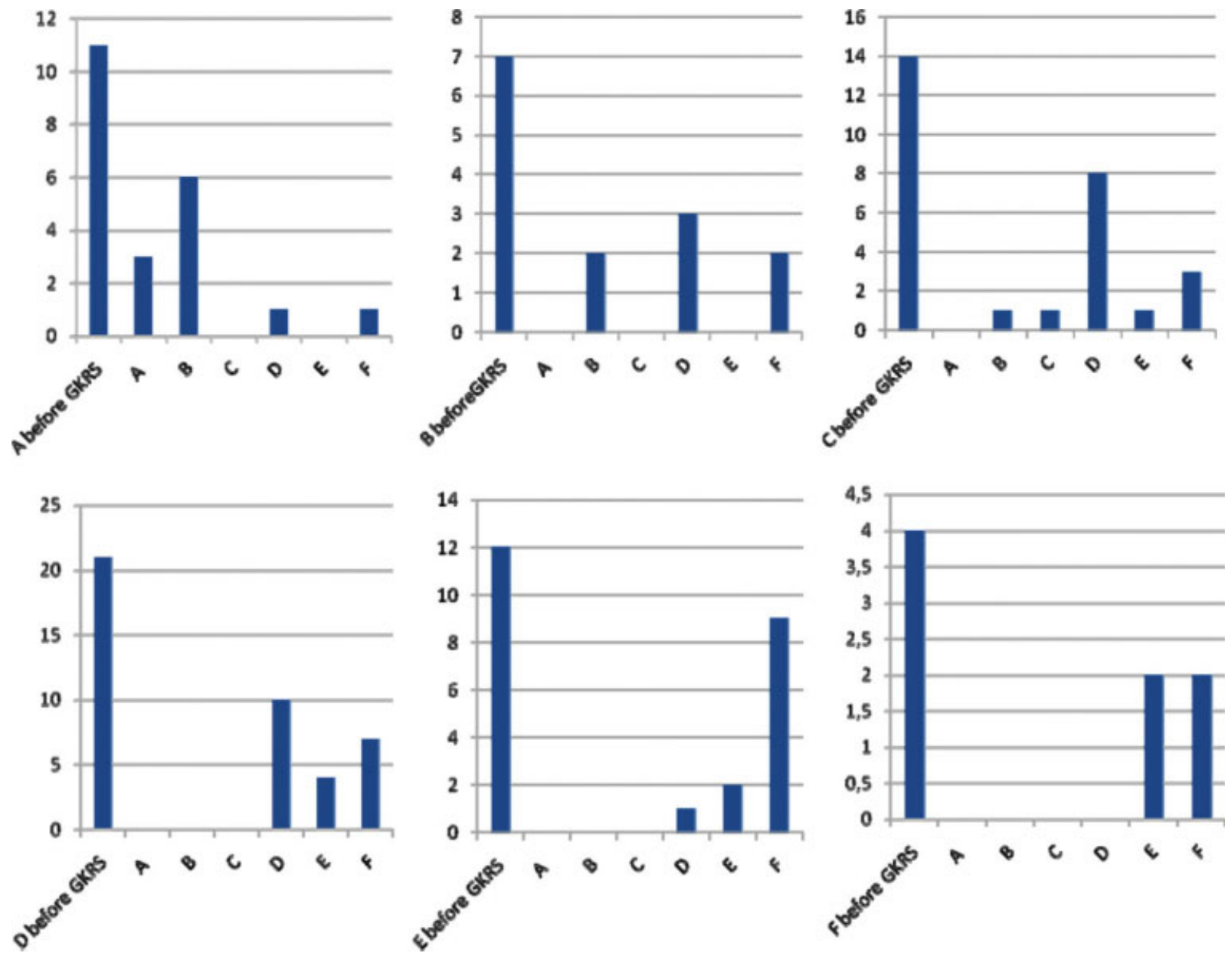

Fig. 2. Hearing classification (Tokyo 2003) before and after gamma knife radiosurgery (GKRS). The number of patients is given per class in each first bar. The black bars indicate the distribution of these patients after GKRS. [Color figure can be viewed in the online issue, which is available at www.interscience.wiley.com.] 


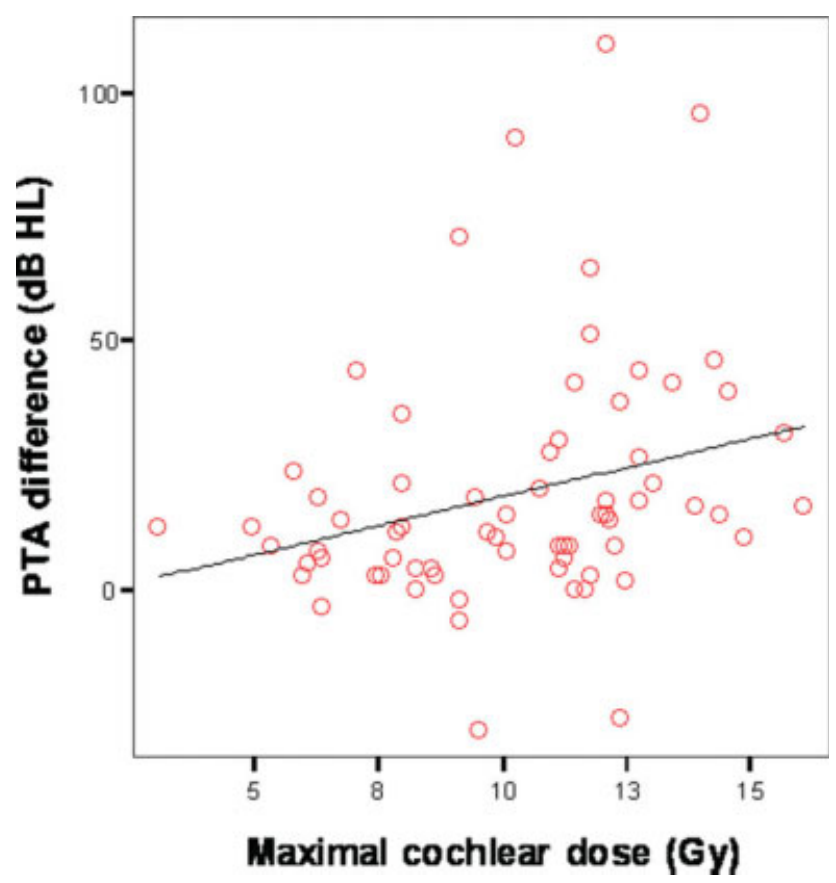

Fig. 3. The correlation between pure tone average (PTA) difference and maximal cochlear dose $(r=0.3, P<.05)$. [Color figure can be viewed in the online issue, which is available at www.interscience. wiley.com.]

when the fundus of the Internal auditory meatus (IAM) is occupied by tumor.

In the present study we found that high PTA before GKRS was correlated with small tumor size, which is difficult to explain in relation to some of the theories

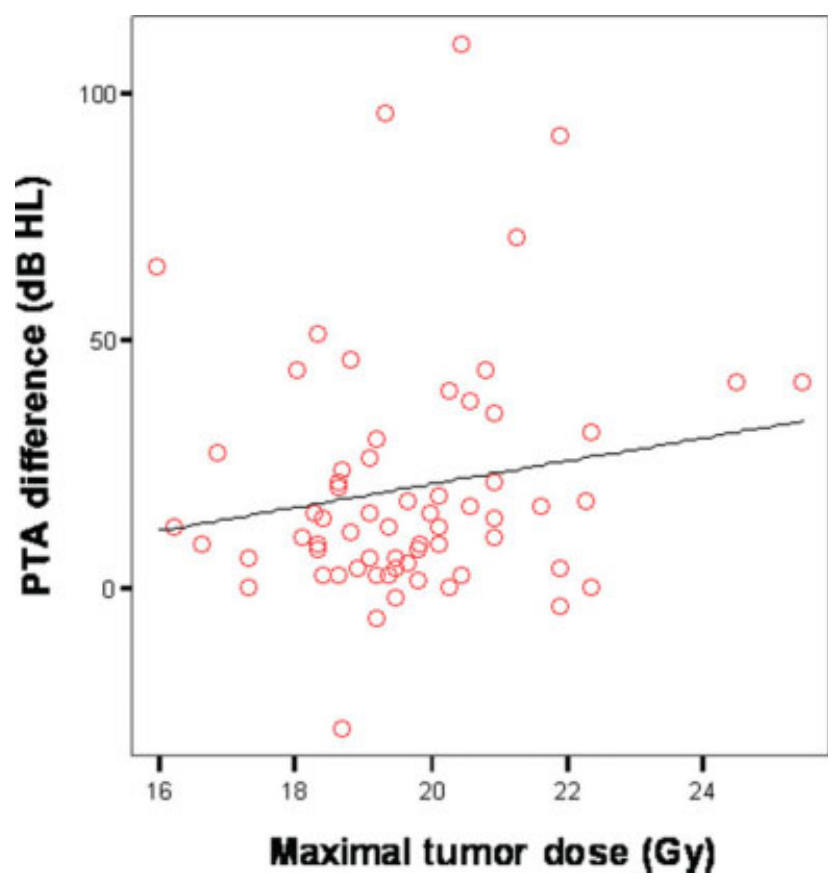

Fig. 4. The correlation between pure tone average (PTA) difference and maximal tumor dose $(r=0.16, P=.2)$. [Color figure can be viewed in the online issue, which is available at www.interscience. wiley.com.]

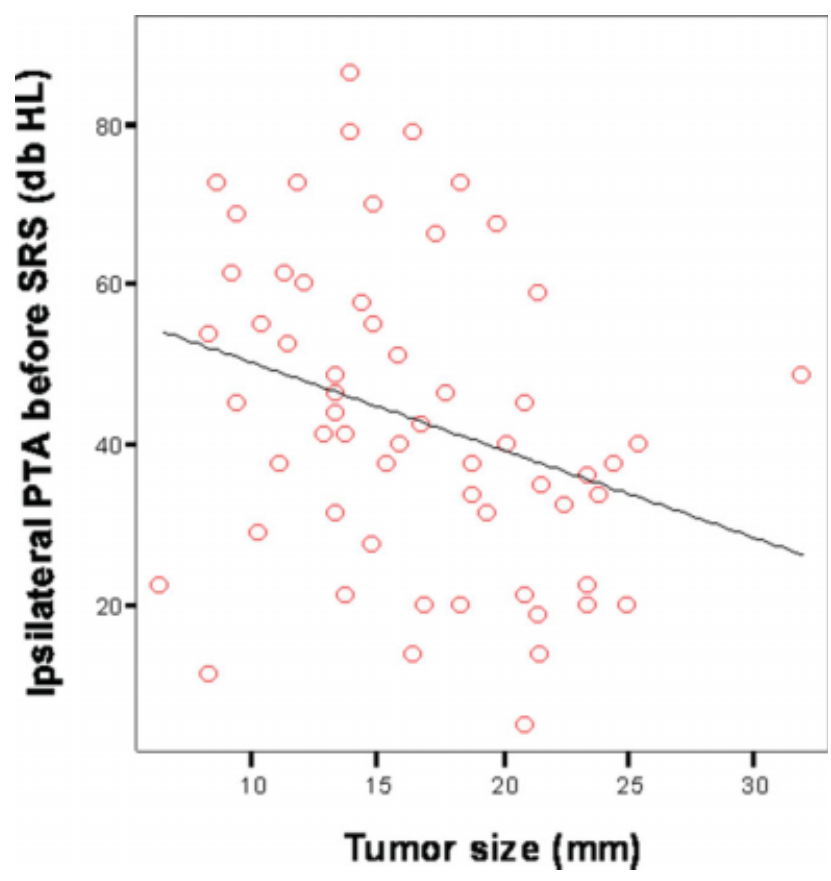

Fig. 5. The correlation between pure tone average (PTA) before gamma knife radiosurgery and tumor size $(r=0.3, P<.05)$. SRS $=$ stereotactic radiosurgery. [Color figure can be viewed in the online issue, which is available at www.interscience.wiley.com.]

mentioned above. A possible explanation could be that some tumors cause hearing loss earlier, for whatever reason, and thereby trigger a visit to a doctor at an early stage.

Over the past decade, a number of authors have addressed hearing preservation. Yet despite the meeting in Tokyo, there is still no consensus on systems for reporting results in VS research. This obviously makes comparison more difficult. To be able to compare our results, we followed the Tokyo guidelines for reporting hearing and tumor size, hoping other authors would do the same. ${ }^{6}$

As the experience with GKRS increased, the dose delivered to the tumor, and especially to the surrounding tissue, has declined. To compare our results, we looked for studies that used a similar radiation dosage.

Paek et al. studied 25 VS patients with serviceable hearing (PTA $<50 \mathrm{~dB}$, SDS $>50 \%$ ) before GKRS. ${ }^{9}$ The mean marginal dose used was $12 \mathrm{~Gy}$. They found a

TABLE VI.

Individual Hearing Classification (Tokyo 2003) Difference Between Before and After GKRS.

\begin{tabular}{lc}
\hline Class Difference & No. \\
\hline-1 & 4 \\
No difference & 19 \\
+1 & 29 \\
+2 & 11 \\
+3 & 1 \\
+4 & 2 \\
+5 & 2 \\
\hline
\end{tabular}

GKRS = gamma knife radiosurgery. 
TABLE VII.

The Mean PTA Difference Between Before and After GKRS Was Significantly Higher in the Group of Patients With an Occupied Fundus $(P<.005)$.

\begin{tabular}{lccc}
\hline & $\begin{array}{c}\text { Mean PTA } \\
\text { Difference } \\
(\mathrm{dB} \mathrm{HL})\end{array}$ & $\begin{array}{c}\text { Standard } \\
\text { Deviation }\end{array}$ & $\begin{array}{c}\text { Standard } \\
\text { Error, } \\
\text { Mean }\end{array}$ \\
\hline Fundus free $(\mathrm{n}=39)$ & 11.3 & 18.1 & 2.9 \\
$\begin{array}{l}\text { Fundus filled with } \\
\text { tumor }(\mathrm{n}=30)\end{array}$ & 29.5 & 28.3 & 5.2 \\
\hline
\end{tabular}

PTA = pure tone average; GKRS = gamma knife radiosurgery; $\mathrm{dB}$ $\mathrm{HL}=$ decibels hearing level.

serviceable hearing preservation of $52 \%$. Chopra et al. studied hearing preservation in a group of 110 patients all with a follow-up $>3$ years. ${ }^{10}$ Their marginal dose was between 12.5 Gy and 13 Gy. They found a serviceable hearing preservation of $77 \%$. The classification serviceable hearing, used in these studies, is comparable to our class A, B, C, and D combined. More in line with Chopra et al., we found a hearing preservation of $68 \%$ in this group of patients (class A, B, C, and D combined) even though our follow-up period was shorter.

Recently, Massager et al. published a paper about the influence of the radiation dose on the cochlea. ${ }^{11}$ In a group of 60 patients with serviceable hearing, 32 patients $(53 \%)$ maintained serviceable hearing after GKRS. In their study a marginal tumor dose of $12 \mathrm{~Gy}$ was prescribed at the $50 \%$ isodose level. As in our study, the maximum dose at the cochlea was determined. The mean maximum radiation dose was 8.51 Gy (3 Gy-15 Gy). In our study we found 10.27 Gy (3 Gy-16 Gy). They also found a strong significant relation $(P=.0005)$ between maximal cochlear dose and hearing preservation, a relation that was even stronger than in our study $(P=.02)$. Their finding is remarkable and indicates the significance of cochlear radiation dose. Unfortunately, they do not discuss their results in light of a possible relation between the dose on the tumor itself and hearing preservation. We could not demonstrate such a correlation in our study group $(P=.2)$.

Gamma knife irradiation techniques were developed to target the radiation effects more precisely so as to avoid collateral damage in healthy tissues. Due to the relatively small distance between tumor and cochlea, and the steep curve of the dose volume histogram, even a slight improvement in pointing out the tumor can have significant effects on the maximum cochlear dose.

Although a longer follow-up period is required to evaluate the long-term results, there is increasing evidence that the first year after the GKRS treatment is an important predictor for later hearing deterioration. ${ }^{9,12,13}$

Due to the setting of this study, it was impossible to standardize the timing of audiometry before and after GKRS. This might have influenced our results. However, due to the meticulous registration and the detailed data set, the results of this study realistically document the impact of GKRS in general, and cochlear dose in particular, on hearing preservation. We hypothesize that it is possible to lower the maximum dose on the cochlea if the radiation planning protocol for VS is further optimized.

\section{CONCLUSION}

Hearing preservation is not correlated to the maximal radiation dose at the tumor but only to the maximal dose at the cochlea. The purpose of developing GKRS techniques was to avoid collateral damage in healthy tissues. This study emphasizes the need for exact radiation planning to reduce the cochlear radiation dose if the hearing is to be preserved.

\section{BIBLIOGRAPHY}

1. Evans DG, Moran A, King A, Saeed S, Gurusinghe N, Ramsden $R$. Incidence of vestibular schwannoma and neurofibromatosis 2 in the North West of England over a 10-year period: higher incidence than previously thought. Otol Neurotol 2005;26:93-97.

2. Tos M, Stangerup SE, Caye-Thomasen P, Tos T, Thomsen J. What is the real incidence of vestibular schwannoma? Arch Otolaryngol Head Neck Surg 2004;130:216-220.

3. Anderson TD, Loevner LA, Bigelow DC, Mirza N. Prevalence of unsuspected acoustic neuroma found by magnetic resonance imaging. Otolaryngol Head Neck Surg 2000; 122:643-646.

4. Leksell L. The stereotaxic method and radiosurgery of the brain. Acta Chir Scand 1951;102:316-319.

5. Leksell L. A note on the treatment of acoustic tumours. Acta Chir Scand 1971;137:763-765.

6. Kanzaki J, Tos M, Sanna M, Moffat DA, Monsell EM, Berliner KI. New and modified reporting systems from the consensus meeting on systems for reporting results in vestibular schwannoma. Otol Neurotol 2003;24:642-648.

7. Committee on Hearing and Equilibrium guidelines for the evaluation of hearing preservation in acoustic neuroma (vestibular schwannoma). American Academy of Otolaryngology-Head and Neck Surgery Foundation, Inc. Otolaryngol Head Neck Surg 1995;113:179-180.

8. Graamans K, Van Dijk JE, Janssen LW. Hearing deterioration in patients with a non-growing vestibular schwannoma. Acta Otolaryngol 2003;123:51-54.

9. Paek SH, Chung HT, Jeong SS, et al. Hearing preservation after gamma knife stereotactic radiosurgery of vestibular schwannoma. Cancer 2005;104:580-590.

10. Chopra R, Kondziolka D, Niranjan A, Lunsford LD, Flickinger JC. Long-term follow-up of acoustic schwannoma radiosurgery with marginal tumor doses of 12 to $13 \mathrm{~Gy}$. Int J Radiat Oncol Biol Phys 2007;68:845-851.

11. Massager N, Nissim O, Delbrouck C, et al. Irradiation of cochlear structures during vestibular schwannoma radiosurgery and associated hearing outcome. $J$ Neurosurg 2007;107:733-739.

12. Hirsch A, Noren G. Audiological findings after stereotactic radiosurgery in acoustic neurinomas. Acta Otolaryngol 1988;106:244-251.

13. Yamasoba T, Kurita H, Ito K, et al. Auditory findings after stereotactic radiosurgery in acoustic neurinoma. Skull Base Surg 1996;6:163-167. 